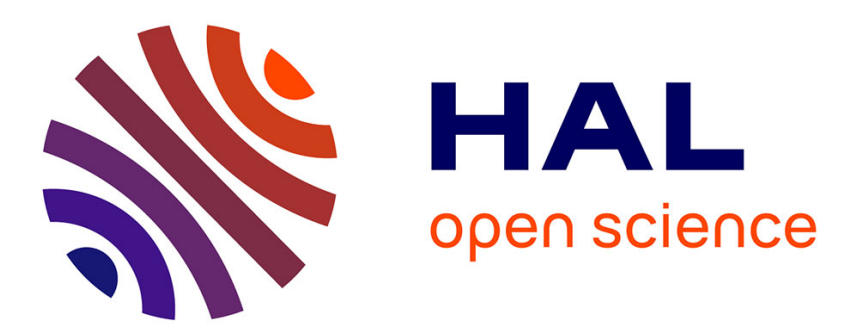

\title{
Sensor-based algorithm for collision-free avoidance of mobile robots in complex dynamic environments
}

\author{
Dimitri Leca, Viviane Cadenat, Thierry Sentenac
}

\section{To cite this version:}

Dimitri Leca, Viviane Cadenat, Thierry Sentenac. Sensor-based algorithm for collision-free avoidance of mobile robots in complex dynamic environments. 2019 European Conference on Mobile Robots (ECMR), Sep 2019, Prague, Czech Republic. p.1-6, 10.1109/ECMR.2019.8870344 . hal-02334205

\section{HAL Id: hal-02334205 https://imt-mines-albi.hal.science/hal-02334205}

Submitted on 2 Mar 2020

HAL is a multi-disciplinary open access archive for the deposit and dissemination of scientific research documents, whether they are published or not. The documents may come from teaching and research institutions in France or abroad, or from public or private research centers.
L'archive ouverte pluridisciplinaire HAL, est destinée au dépôt et à la diffusion de documents scientifiques de niveau recherche, publiés ou non, émanant des établissements d'enseignement et de recherche français ou étrangers, des laboratoires publics ou privés. 


\title{
Sensor-based algorithm for collision-free avoidance of mobile robots in complex dynamic environments
}

\author{
D. Leca ${ }^{1,2}$, V. Cadenat ${ }^{1,2}$, T. Sentenac ${ }^{1,3}$
}

\begin{abstract}
This paper deals with the problem of navigation of unmanned vehicles through poorly known environments cluttered with static and dynamic obstacles. The robot is equipped with a LiDAR able to provide a scan of the surroundings and with classical dedicated localization sensors (odometry, IMU). The proposed navigation strategy relies on: (i) a new concept called Enhanced Laser Scan (ELS), which is built from the current laser scan by adding virtual points along the predicted trajectory of the obstacles ; (ii) two sensor-based controllers allowing respectively to reach the goal and to avoid obstacles. These controllers relying on the richer information provided by the ESL, they will be able to anticipate and safely avoid both static and moving obstacles ; (iii) a high-level decision process allowing a better choice of the sense-of-motion (SOM) around the obstacle and its reassessment if needed.
\end{abstract}

\section{INTRODUCTION}

In mobile robotics, one of the key feature is the ability for the robot to safely and smoothly navigate through an unknown environment. In this environment, the robot will encounter static as well as dynamic obstacles. If an extensive amount of papers has been published about how to deal with static obstacles [1], the case of dynamic obstacles is still a discussed issue [2]. Indeed, in an unknown environment, few assumptions can be made about the obstacles lying in the robot vicinity. These obstacles can be rigid or with changing shapes. Their trajectory and velocity can be subject to unpredictible changes with time. In these cases, global planners are limited [1], since they require a previous knowledge of the environment. Because of this minimal representation, local approaches appear to be more suitable, since they will allow the robot to reactively adapt to any change in its environment. They can be split in two categories: local planners, that use the local information provided by the sensors to compute a short-horizon trajectory, and reactive controllers, that directly generate the current control using the current provided information.

Among local planners that take into account dynamic obstacles, the well-known Dynamic Window Approach (DWA) has been generalized in [3] and [4] to handle moving obtacles. The concept of Velocity Obstacles (VO) chooses among all possible velocities the one that guarantees noncollision. This method has been extended to handle dynamic obstacles, with the Acceleration-Velocity Obstacles [5] or the Non-Linear Velocity Obstacles [6], [7]. However, all of

\footnotetext{
${ }^{1}$ CNRS, LAAS, 7 avenue du colonel Roche, F-31400 Toulouse, France

${ }^{2}$ Univ de Toulouse, UPS, LAAS, F-31400, Toulouse, France

${ }^{3}$ Institut Clément Ader (ICA), Université de Toulouse, CNRS, Mines Albi, UPS, INSA, ISAE-SUPAERO, Campus Jarlard, F-81013 Albi CT Cedex 09, France
}

these methods assume that the encountered obstacles are small and cylindrical to take into account their velocities. The methods based on Artificial Potential Fields have also been widely investigated to deal with mobile obstacles. They suffer from well-known drawbacks, such as local minima [8]. As local planners, their adaptation to dynamic environments are often performed under strong assumptions, such as: the precise knowledge of the velocity and the acceleration of each obstacle, their size and shape, or the number to be avoided simultaneously [9], [10], [11], [12]. Another class of methods, called bug methods, consists in the following reasoning: moving toward the goal following a straight line, switching to an avoidance method if a collision threat occurs, and resuming to a straight line toward the goal once the obstacle is avoided. These methods require few assumptions and knowledge about the environment. They have been successfully applied to different kinds of robots in [13], [14], [15], and have dealt with complex scenes involving numerous obstacles whose shapes and movements have evolved during the mission.

In this work, we assume that the environment is poorly known: no map is provided prior to the navigation and no information about the position, shape or motions of the possibly encountered obstacles is available. The robot is equipped with a LiDAR able to provide a scan of the surroundings. In such a case, following our previous study [16], local reactive methods have been shown to be more adapted. It is proposed such a navigation strategy based on two sensorbased controllers allowing respectively to reach the goal and to avoid static obstacles. Here, the presented strategy extends our previous works on that topic [17], [18], [16]. In this set of works, the avoidance technique relies on the definition of a spiral [19] as first proposed in [20] and [21] for UAV (Unmanned Aerial Vehicles). If our most recent strategy developed in [16] allows to safely and efficiently navigate through a static environment cluttered with various shaped obstacles, it is still impossible to deal with dynamic ones. This paper allows to overcome this limitation by proposing an enhanced navigation strategy able to avoid both static and dynamic objects whose both motion and shape are not a priori known. Three main improvements have been made with respect to [16]. At low level, the points provided by the LIDAR have been treated so that: (i) static and dynamic objects can be separated ; (ii) a prediction of the moving objects velocity is performed; (iii) this prediction is used to add to the current LIDAR scan a set of virtual points representing each obstacle predicted motion. This allows to produce an 'enhanced laser scan' (ELS) which will be 
used to feed the avoidance controller designed in [16]. The same control law is then applied to the robot but, thanks to the richer information contained in the ELS, it becomes possible to anticipate the motion of mobile obstacles. The second improvement with respect to [16] is a high level one. It concerns the choice of the sense of motion (SOM) around the obstacle. The SOM decision takes into account the motion of the obstacle and can be reassessed if this latter happens to suddenly modify its trajectory. This allows to avoid undesirable behaviors where the robot could be dragged away by an object moving at the same speed. Finally, a linear velocity profile has been designed to improve the robot behavior in the obstacle vicinity.

The paper is organized as follows. Section II introduces the spiral model, its parameters, and the control laws designed to follow the spirals. Section III introduces the algorithms designed to take into account dynamic parts of the environment. Section IV deals with the implemented decision process while section $\mathrm{V}$ presents simulation results showing the interest of the approach.

\section{Preliminaries}

This section deals with the preliminaries of the spiral avoidance technique [16].

\section{A. Spiral modelling}

The section focuses on parameters used to define the equiangular spiral. As shown in figure 1a, the spiral is described by the point $O_{p}$ moving on a plane with respect to a fixed point $O_{s}$. This point is considered as the center of the spiral. $\overrightarrow{v^{*}}$ is the velocity vector applied to $O_{p}$ and its norm is denoted $v^{*}(t)$. Moreover $\overrightarrow{d^{*}}$ is the vector connecting $O_{s}$ to $O_{p}$ whose norm is $d^{*}(t)$. Finally $\alpha^{*}(t)$ is defined as the oriented angle between $\overrightarrow{v^{*}}$ and $\overrightarrow{d^{*}}$. In [19] it is shown that if both $v^{*}(t)$ and $\alpha^{*}(t)$ are constant then $O_{p}$ describes a spiral whose center is $O_{s}$. They are then respectively denoted by $v^{*}$ and $\alpha^{*}$ in the sequel. Moreover [19] states that $\dot{d}^{*}(t)=-v^{*} \cos \left(\alpha^{*}\right)$. Thus the executed spiral only depends on the value of $\alpha^{*}$. Its sign allows to define the SOM (clockwise if negative, anticlockwise if positive), while its value fixes the type of spiral: inward if $\left|\alpha^{*}\right|<\pi / 2$, outward if $\pi / 2<\left|\alpha^{*}\right| \leq \pi$, circle if $\alpha^{*}= \pm \pi / 2$. From this analysis, it follows that this concept can be easily adapted to perform an obstacle avoidance motion. Indeed, fixing the spiral center point (SCP) on the obstacle surface and selecting a suitable couple of $\alpha^{*}$ and $d^{*}$ allows us first to choose the SOM for the avoidance around the obstacle, and then to control the desired distance $d^{*}$ and its evolution. This paper states how to choose these parameters from the available sensory data to perform an efficient and safe avoidance motion.

\section{B. Robot modelling}

The robot has four-wheel skid steering drive, which allows the robot to turn on itself. Its model is presented in figure $1 \mathrm{~b}$. $F_{w}=\left(O_{w}, \overrightarrow{x_{w}}, \overrightarrow{y_{w}}, \overrightarrow{z_{w}}\right)$ is the frame linked to the world, while $F_{r}=\left(O_{r}, \overrightarrow{x_{r}}, \overrightarrow{y_{r}}, \overrightarrow{z_{r}}\right)$ is the frame attached to the robot. $\chi(t)=[x(t), y(t), \theta(t)]^{T}$ represents the pose of the

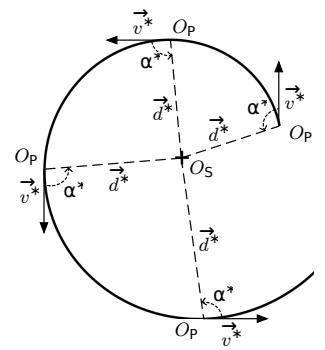

(a) Spiral model

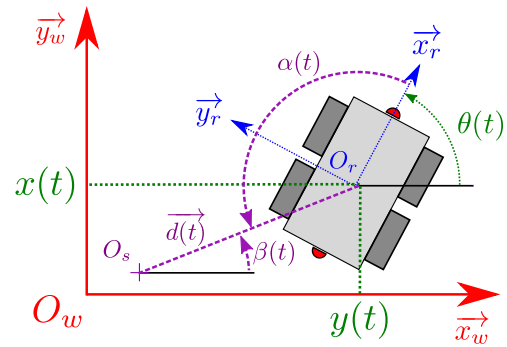

(b) Robot model
Fig. 1: Spiral and robot modelling

robot in the world frame, where $x(t)$ and $y(t)$ are the coordinates of $O_{r}$ in $F_{w}$ and $\theta(t)$ is the angle between $\overrightarrow{x_{w}}$ and $\overrightarrow{x_{r}} . O_{s}$ is the center of the spiral to be followed, while $\vec{d}$ represents the vector connecting $O_{s}$ to $O_{r}$ and $\alpha(t)$ is the angle between $\overrightarrow{x_{r}}$ and $\vec{d} . \beta(t)$ is the angle between $\overrightarrow{x_{w}}$ and $\vec{d}$. Knowing that $\alpha(t)=\pi-\theta(t)+\beta(t)$, it should be noticed that:

$$
\dot{\alpha}(t)=-\dot{\theta}(t)+\dot{\beta}(t)=-\omega(t)+\frac{v(t)}{d(t)} \sin (\alpha(t))
$$

Consequently, $d(t)$ represents the distance between $O_{s}$ and $O_{r}$. Following [19] : $\dot{d}(t)=-v(t) \cos (\alpha(t))$.

\section{Obstacle avoidance controllers design}

To perform the avoidance, the robot must first reach the desired spiral, and then follow it around the obstacle. Two controllers have been proposed in [16].

1) Definition of the errors: The two following errors must be vanished: $e_{\alpha}=\alpha(t)-\alpha^{*}$ and $e_{d}=d(t)-d^{*}(t)$. In [18], in order to track a spiral defined by its SCP together with $v^{*}$ and $\alpha^{*}$, it is proposed to impose $v(t)=v^{*} \neq 0$.

2) First controller design: The following control law $\omega_{A}$ allows to make the errors $e_{\alpha}$ and $e_{d}$ converge toward zero asymptotically thanks to an exact input to state linearization method [22]:

$$
\omega_{A}(t)=\frac{\lambda_{1} e_{d}(t)+\lambda_{2} \dot{e}_{d}(t)}{v^{\star} \sin \left(e_{\alpha}(t)+\alpha^{\star}\right)}+\frac{v^{\star} \sin \left(e_{\alpha}(t)+\alpha^{\star}\right)}{e_{d}(t)+d^{\star}(t)}
$$

This controller maintains the robot along the desired spiral, with $\lambda_{1}, \lambda_{2}>0$, but suffers from singularities when $e_{\alpha}(t)+$ $\alpha^{*}=\alpha(t)=k \pi, k \in \mathbb{Z}$.

3) Second controller design: This controller is intended to avoid this latter problem. Following [18], an hybrid error is introduced: $e_{S}(t)=e_{\alpha}(t)-\epsilon(t) \alpha_{D}$, where $\epsilon(t)$ is the normalized error between $d^{*}(t)$ and $d(t)$, saturated to \pm 1 :

$$
\epsilon(t)=\operatorname{sign}\left(d^{*}(t)-d(t)\right) \frac{\min \left(\left\|d^{*}(t)-d(t)\right\|, n\right)}{n}
$$

where $n \in \mathbb{N}^{*}$. Additionally, $\alpha_{D}$ is defined as $\operatorname{sign}\left(\alpha^{*}\right) * \pi-$ $\alpha^{*}$ if $d^{*}(0)>d(0)$, or $\alpha^{*}$ if $d^{*}(0)<d(0)$. To make $e_{S}(t)$ vanish, the following controller $\omega_{B}$ is proposed in [18].

$$
\omega_{B}(t)=\lambda_{S} e_{S}(t)+\frac{v(t)}{d(t)} \sin (\alpha(t))-\dot{\alpha}_{S}(t, d)
$$

where $\lambda_{S}>0$. As shown in [18], this controller is locally asymptotically stable, once $\alpha(t)$ overpasses $\alpha^{*}(t)$. 
4) Switching strategy: To overcome the singularity problem which occurs when $\alpha(t)$ vanishes, it is proposed to switch between $\omega_{A}$ and $\omega_{B}$. When the error $e_{\alpha}(t)$ drops below a threshold $e_{\alpha}^{\text {switch }}$, the $\omega_{A}(t)$ controller is sent to the robot. Else, $\omega_{B}(t)$ is sent. To obtain the final control law $\omega(t)$, a sliding-window smoothening is applied to avoid discontinuities in the control inputs when switching between $\omega_{A}$ and $\omega_{B}$.

\section{Spiral-based navigation strategy for static environments}

1) Choice of $\alpha^{*}$ and $d^{*}$ : In [16], an angle of $\alpha^{*}= \pm \pi / 2$ is imposed. Its sign depending on the SOM around the obstacle. Consequently, $d^{*}$ is constant.

2) Choice of the SCP: After a LiDAR acquisition around the robot, the closest point $O_{c}$ from the robot is computed. Then, all the LiDAR points within a $2 d^{*}$ meters radius circle centered on $O_{c}$ are used to compute their barycenter $O_{b}$. Finally, $O_{c}$ and $O_{b}$ are compared, and the closest one to the robot is chosen as the spiral center point $O_{s}$.

3) Go-to-Goal controller and switching conditions: The Go-To-Goal (GTG) controller can be defined by any controller allowing to reach the goal. Here, we have chosen a basic proportional controller correcting the heading of the robot to reach $O_{g}$. As explained in [16], the switch between the avoidance controller and the GTG controller occurs if an obstacle lies within a half-circle heading toward the goal, whose radius is between $d^{*}$ and $2 d^{*}$ depending on the relative orientation between the robot and the obstacle. Thus, if an obstacle lays in front of the robot, the avoidance will be triggered earlier.

This section has been devoted to a brief recalling of the navigation strategy described in [16]. This method has been proven to be efficient for static environments but cannot deal with mobile obstacles. This paper extends these works by proposing three improvements: (i) the design of an enhanced laser scan (ELS) containing the current laser points and the virtual points modelling the mobile obstacle motion; (ii) the possible reassessment of the SOM to react adequately with respect to obstacle motion; and (iii) the design of a safer linear velocity profile. Thanks to these improvements, the same control law can be used for both static and dynamic environments, generalizing our previous works.

\section{ENHANCED LASER SCAN}

This section focuses on our first contribution, the computation of the dynamics of each point detected by the LiDARs, and the generation of the artificial laser points. The aim of these virtual points is to model the expected evolution of the dynamic parts of the environment. These virtual points will be merged with the initial laser scan to provide an enhanced laser scan (ELS) that will feed the algorithm presented in part II. Using the ELS, the robot will anticipate the evolution of each moving obstacle, taking safer decisions such as an earlier avoidance trigger or a more suitable SOM.

\section{A. Detection of the moving points}

We denote by $R_{p}$ and $R_{c}$ the frames attached to the robot at time $t_{p}$ and $t_{c}$, with $t_{p}<t_{c}$. We also introduce $P_{R_{c}}(t)$ and $P_{R_{p}}(t)$ the arrays of laser point coordinates taken at time $t$, given in the robot frames $R_{p}$ and $R_{c}$. The algorithm works by comparing the points from the current laser scan $P_{R_{c}}\left(t_{c}\right)$ taken at time $t_{c}$ with the points from a previous laser scan $P_{R_{p}}\left(t_{p}\right)$. The transformation matrix $R_{c} T_{R_{p}}$ between $R_{p}$ and $R_{c}$ can be obtained using local localization methods. Thus, the coordinates of the points from the previous acquisition in the current robot frame $R_{c}$ can be computed such as:

$$
P_{R_{c}}\left(t_{p}\right)={ }^{R_{c}} T_{R_{p}} \cdot P_{R_{p}}\left(t_{p}\right)
$$

Algorithm 1 finds for each point in $P_{R_{c}}\left(t_{c}\right)$ its closest point in $P_{R_{c}}\left(t_{c}\right)$. If this distance is over a threshold $D$, the point is considered as moving. Else, it is not. The algorithm produces two output matrices, $\widetilde{P}_{R_{c}}\left(t_{c}\right)$ and $\widetilde{P}_{R_{c}}\left(t_{p}\right)$. These matrices respectively contains the subset of points from $P_{R_{c}}\left(t_{c}\right)$ and $P_{R_{c}}\left(t_{p}\right)$ that are seen to be moving, and will be used to characterize the robot environment.

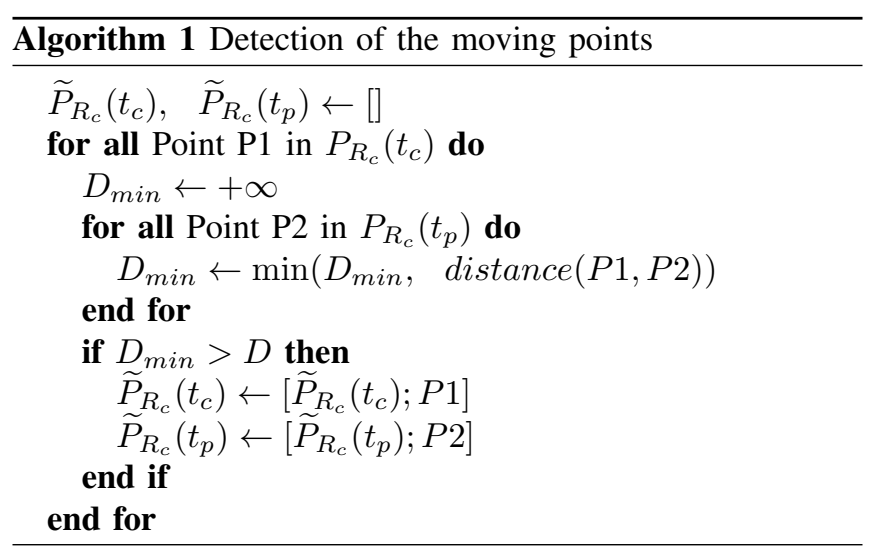

\section{B. Characterization of the obstacles}

1) Obstacle clustering: $\underset{\widetilde{P}}{\mathrm{~A}}$ spatial clustering is operated on the points contained in $\widetilde{P}_{R_{c}}\left(t_{c}\right)$. Since the obstacles are well-separated, any well-known clustering technique, such as hierarchical clustering [23] can be used. This allows to be able to handle any number of obstacles.

2) Obstacle velocity vector computation: After the clustering, the velocity vector of each obstacle is estimated. For each cluster, the barycenters $B_{R_{c}}\left(t_{c}\right)$ and $B_{R_{c}}\left(t_{p}\right)$ of their corresponding points in $\widetilde{P}_{R_{c}}\left(t_{c}\right)$ and $\widetilde{P}_{R_{c}}\left(t_{p}\right)$ are computed. The velocity vector for this cluster is obtained as follows:

$$
\vec{V}_{R_{c}}\left(t_{c}\right)=\left(\begin{array}{l}
v_{x}\left(t_{c}\right) \\
v_{y}\left(t_{c}\right)
\end{array}\right)_{R_{c}}=\frac{B_{R_{c}}\left(t_{c}\right)-B_{R_{c}}\left(t_{p}\right)}{t_{c}-t_{p}}
$$

\section{Projection of the virtual points}

Using the velocity vector, the points that will be added to the ELS are computed. 
1) Horizon: It has been seen that the avoidance can be triggered at a maximal distance of $2 d^{*}$. Depending on its velocity $v(t)$, the robot could reach this distance in $2 d^{*} / v(t)$ seconds. We then define $t_{h}=2 d^{*} / v_{\text {max }}$. At any time when the avoidance could be triggered, it is necessary to take into account where any moving obstacles might be in $t_{h}$ seconds. Consequently, depending on its cluster, each moving point will be projected along the vector $\vec{d}_{h}=\vec{V}_{R_{c}}\left(t_{c}\right) \cdot t_{h}$.

2) Projection: For each moving obstacle, virtual points are added to the initial laser scan to model the future trajectory of this obstacle. The method consists in translating, for each cluster, the points from a given distance along the velocity vector, and to add a hull linking the initial points and the translated points. Furthermore, in order to prevent the angular control law to unexpectedly reach too high values, no virtual point should be added within a $d^{*}$ perimeter around the robot. Figure 2 shows how the virtual points are

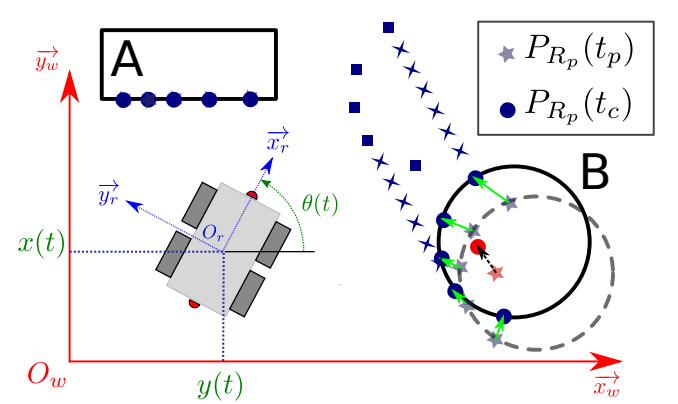

Fig. 2: Computation process of the dynamic of the obstacles and the virtual points.

computed. The environment is cluttered with two obstacles. One rectangular static obstacle (A), and one circular dynamic obstacle (B). The solid lines represent both obstacles at $t=t_{c}$. The solid blue points are the points $P_{R_{c}}\left(t_{c}\right)$ currently detected by the LiDAR. The dotted circle represents the (B) obstacle as it was at time $t=t_{p}$, while the grey star points are the points $P_{R_{c}}\left(t_{p}\right)$, that were detected at the previous time $t_{p}$, projected in the current robot frame $R_{c}$. At the beginning of the algorithm, each point from $P_{R_{c}}\left(t_{p}\right)$ is matched with its closest point in $P_{R_{c}}\left(t_{c}\right)$, represented by the green arrows. If the distance between these matched points is bigger than a threshold, these points are considered to be moving. This is the case of the points lying in the circle, but not the case of those belonging to the static obstacle (A). At the end of this process, $\widetilde{P}_{R_{c}}\left(t_{p}\right)$ contains the five grey star points, $\widetilde{P}_{R_{c}}\left(t_{c}\right)$ the five blue points lying in the circle. Then, the clustering is performed, and the barycenters $B_{R_{c}}\left(t_{c}\right)$ and $B_{R_{c}}\left(t_{p}\right)$ are computed, represented respectively by the red circle point and the red star point. $\vec{V}_{R_{c}}\left(t_{c}\right)$ is then calculated, represented by the black arrow between the red points. This process being done, the virtual points can be computed and added to the final scan. The five blue round points are translated along their respective vector $\overrightarrow{d_{h}}$, generating the five squared blue points. The hull is then created, producing the blue star points.

\section{NAVIGATION STRATEGY}

\section{A. Sense of motion computation}

For static obstacles, the condition is based on the sign of $\alpha_{c}$ : if $\alpha_{c} \leq 0$, select a clockwise SOM. Else, select a counter-clockwise SOM. For a dynamic environment, the future position of the obstacle must be taken into account at the decision instant. Typically, if the obstacle is at the right of the robot, and moving from the right to the left, the previous computation would produce a clockwise SOM. Such a SOM would make the robot trajectory to cross the obstacle expected path, leading to a collision threat. The proposed algorithm anticipates the motion of dynamic obstacle. When an obtacle is detected and the switch between the GTG controller and the SA controller is triggered, the SOM is determined using $O_{c}$ and its associated velocity vector $\vec{V}_{R_{c}}(t)=\left(\begin{array}{l}v_{x}(t) \\ v_{y}(t)\end{array}\right)_{R_{c}}$.

- If $v_{y}=0$, apply the conditions for static obstacles.

- Else if $v_{y}>0$, select a counter-clockwise SOM. It means that the obstacle is going from right to left.

- Else if $v_{y}<0$, select a clockwise SOM. It means that the obstacle is going from left to right.

\section{B. SOM reassessment}

In complex cases (multiple obstacles, unpredictable obstacle dynamics), it might happen that the SOM computed when the avoidance process was first triggered makes the robot be dragged away by an obstacle moving at the same speed than the robot. Consequently, it is necessary to detect these situations and reassess the SOM whenever needed. These cases can be detected by checking if $v_{x}>0$ and $\left|v_{y}\right|<v_{y}^{0}$. If these conditions are fullfilled, the robot is going in the same direction than the obstacle. If this case occurs, the algorithm reassesses the SOM. Furthermore, at each iteration, the distance between two successive SCP is computed. If this distance is greater than a given threshold, the new SCP belongs to a new obstacle. In this case, the $\mathrm{SOM}$ is also reassessed.

\section{Linear velocity}

In [16], the robot was operating at the same constant linear velocity $v$. For a safer behavior, the robot linear velocity $v$ is mapped with $e_{\alpha}(t)$, to evolve between a maximum velocity $v_{\max }$ and a minimum velocity $v_{\min }$ :

$$
v(t)=v_{\max }-\left(v_{\max }-v_{\min }\right) \frac{\left|e_{\alpha(t)}\right|}{\pi / 2}
$$

This formula leads to a safer behavior. When an obstacle lies in front of the robot $\left(\left|e_{\alpha(t)}\right| \simeq \pi / 2\right)$, the velocity will be minimal. If it is on the side of the robot $\left(\left|e_{\alpha(t)}\right| \simeq 0\right)$, a maximal linear velocity will be produced.

\section{Simulation AND RESUlts}

\section{A. Simulation description}

We have evaluated our method using Matlab software. Starting from its initial pose $O_{r}(0)=O_{w}$, it has to reach a goal $O_{g}$ whose coordinates in the initial robot frame are 


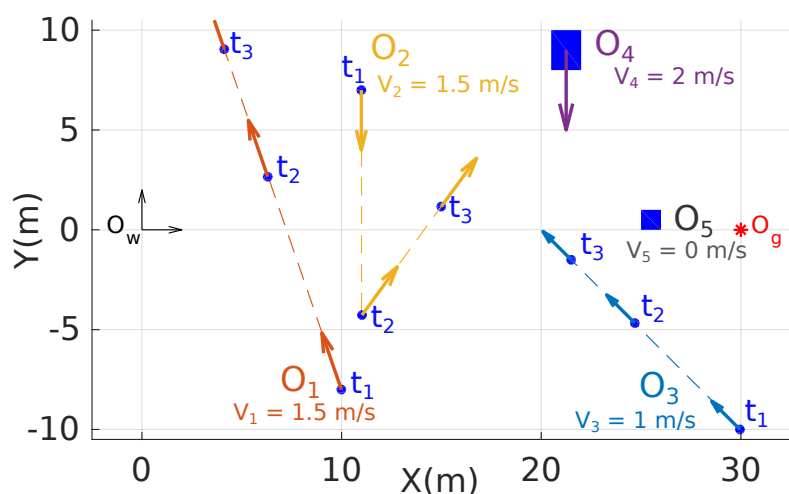

Fig. 3: Positions and movements of the obstacles at three key moments

$\left[x_{g}, y_{g}\right]=[30,0]$. Between the robot and the goal lie several obstacles. Figure 3 shows the position and the velocity of the obstacles at $t_{1}=0 \mathrm{~s}, t_{2}=7.5 \mathrm{~s}$ and $t_{3}=12 \mathrm{~s} . O_{1}$, $\mathrm{O}_{2}$ and $\mathrm{O}_{3}$ are three small convex obstacles, which could represent humans walking. $O_{2}$ changes its trajectory at $t_{2}$. $\mathrm{O}_{4}$ is a car-sized obstacle, and starts moving at $t_{3} . \mathrm{O}_{5}$ is a static obstacle. This environment aims at simulating a typical human environment. The robot linear velocity $v(t)$ evolves between $v_{\min }=0.5 \mathrm{~ms}^{-1}$ and $v_{\max }=1.5 \mathrm{~ms}^{-1}$. To ensure a safe avoidance motion, $d^{*}=3 \mathrm{~m}$. For the controllers, the gains are set as: $\lambda_{1}=0.2, \lambda_{2}=0.2$ and $\lambda_{S}=0.5 \mathrm{~ms}^{-1} . e_{\alpha}^{\text {switch }}=\pi / 12, n=5$, and $v_{y}^{0}=0.5 \mathrm{~ms}^{-1}$. An additive white gaussian noise with $\sigma=0.03$ is applied to the LiDAR distance output. The algorithm runs with a rate time $T_{s}=0.02 \mathrm{~s}$. Furthermore, the interval between $t_{c}$ and $t_{p}$ is chosen at 10 iterations, hence $t_{c}-t_{p}=0.2 s$. To smoothen the control, an averaging sliding window of 5 iterations is applied.

\section{B. Results}

The robot succeeds in reaching its goal in 28.2 seconds, safely avoiding all the obstacles. Figure 4 displays the pose of the robot and its trajectory at four key moments. Red points represent virtual laser points added to the scan. They can be compared with the real obstacle velocities shown on figure 3 and represented by arrows. It can be seen that at each iteration, the virtual laser points are added along the expected future path of each obstacle. The black dots represent the current SCP. Figure 5 displays the linear velocity $v(t)$ and the angular velocity $\omega(t)$ as well as the type of used controllers. Figure 6 presents the evolution of $e_{d}(t)$ and $e_{\alpha}(t)$, as well as $d(t)$ and $d_{c}(t)$, that represents the closest physical point to the robot.

1) Avoidance of $O_{1}$ : From $t=0 \mathrm{~s}$ to $t=1 \mathrm{~s}$, the robot uses the GtG controller and goes toward the goal. $O_{1}$ and $\mathrm{O}_{2}$ are within LiDAR detection range. The algorithm detects that these obstacles are moving, and adds virtual points along their future predicted trajectories. The closest point $O_{c}$ is computed and belongs to the virtual points added from $O_{1}$. This point being within the avoidance trigger range, the robot switches from the GtG controller to the SA controller. At this point, thanks to the obstacle velocity vector, the algorithm detects that the obstacle is going from right to left, and decides a c.c.w. SOM, as it can be seen on figure $4 \mathrm{a}$.

2) Avoidance of $\mathrm{O}_{2}$ : At $t=6 \mathrm{~s}$, the SCP becomes the closest point $\mathrm{O}_{c}$ which is a virtual point derivated from $\mathrm{O}_{2}$. Such a jump in the SCP position triggers the reassessment of the SOM. Because $\mathrm{O}_{2}$ goes from left to right, a c.w. sense of motion is chosen (Figure 4b). As shown in figure 3, $\mathrm{O}_{2}$ suddenly changes its trajectory at $t=7.5 \mathrm{~s}$. Because of its initial choice of SOM, the robot was trying to avoid it in the same direction it is now going. The condition presented in IV-B is met, and the SOM is reassesed, leading to a new c.c.w. sense of motion, as shown on Figures 4c. and 4d. A pike in the angular control output $\omega(t)$ can be observed on Figure 5, corresponding to the SOM changing.

3) Avoidance of $\mathrm{O}_{3}$ and $\mathrm{O}_{4}$ : At $t=16 \mathrm{~s}$, the obstacle $\mathrm{O}_{3}$, which is moving from the right to the left with respect to the robot. A c.c.w. SOM is fixed. Soon after, $\mathrm{O}_{4}$ becomes the closest detected obstacle. This jump in the SCP leads to a reassessment of the SOM, chosen as c.w., since the obstacle is moving from left to right.

4) Avoidance of $O_{5}$ : At $t=20 \mathrm{~s}$, the mobile obstacle $\mathrm{O}_{4}$ has been avoided, and the robot arrives in front of the static objet $O_{5}$. A c.c.w. SOM is chosen. At $t=28.2 \mathrm{~s}$, the simulation ends with the robot reaching its goal.

This simulation highlights the advantages of our method. Using the information about environment provided by the enhanced laser scan, the robot is able to take better decisions about the SOM, leading to a quicker and safer avoidance. Moreover, it can also handle a sudden modification of the obstacles direction by switching its SOM. It can be seen on figure 6 that in spite of the numerous dynamic obstacles, no collision occurs. The controls $v(t)$ and $\omega(t)$ also remain continuous and within acceptable ranges.

\section{CONCLUSION}

This article has presented a novel strategy allowing a mobile robot to navigate through a dynamic environment. It extends previous works based on the spiral avoidance method. It combines the control laws proposed in [16] with a novel method called enhanced laser scan, where virtual points are added to the laser scan. A new decision layer has also been added to improve the choice of the sense-ofmotion. This complete navigation strategy has been tested on Matlab software, and has been proven to be efficient. Therefore, our next step is to implement it on the Air-Cobot plateform in LAAS.

\section{REFERENCES}

[1] H. Choset, K. M. Lynch, S. Hutchinson, G. Kantor, W. Burgard, L. E. Kavraki, and S. Thrun, Principles of Robot Motion, 2005.

[2] M. Hoy, A. S. Matveev, and A. Savkin, "Algorithms for collisionfree navigation of mobile robots in complex cluttered environments: A survey," Robotica, vol. 33, pp. 1-35, 032014.

[3] M. Seder and I. Petrovic, "Dynamic window based approach to mobile robot motion control in the presence of moving obstacles," in Proceedings 2007 IEEE International Conference on Robotics and Automation, April 2007, pp. 1986-1991. 


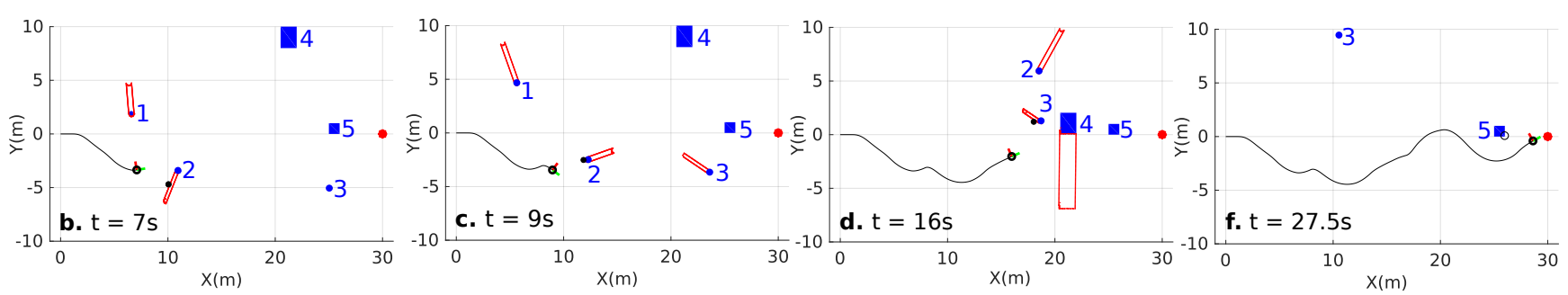

Fig. 4: Obstacle position and robot position and trajectory at different times

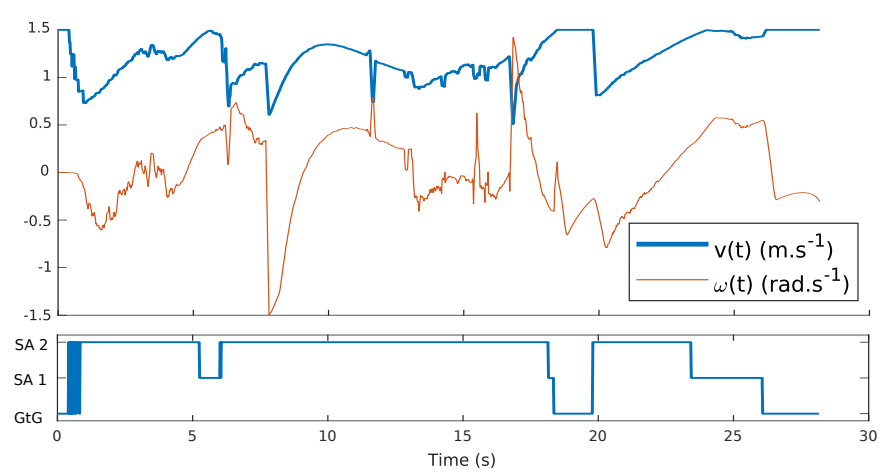

Fig. 5: Linear velocity (in $m s^{-1}$ ), angular velocity (in rad. $\left.s^{-1}\right)$ and controller used (GtG, SA1, or SA2)

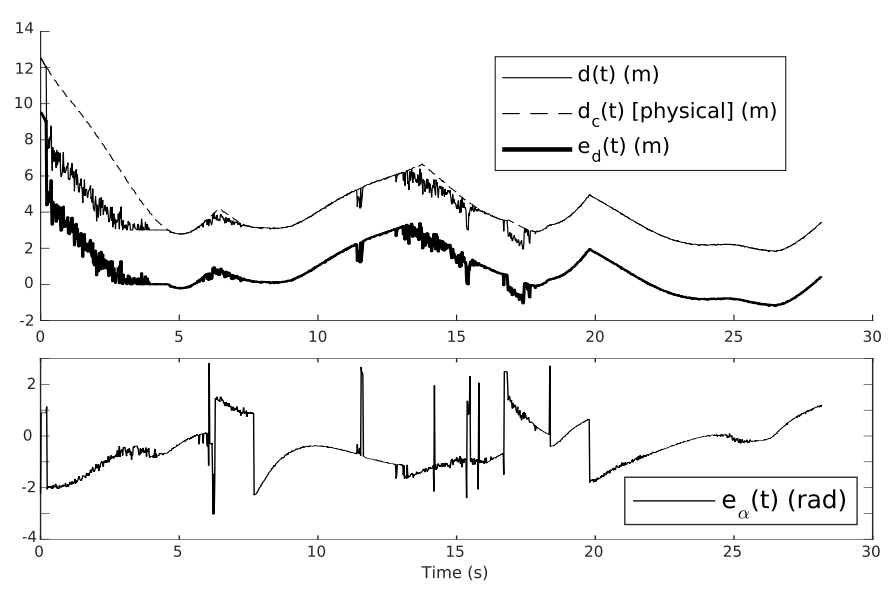

Fig. 6: Distance error $e_{d}(t)$, angular error $e_{\alpha}(t)$, distance to the SCP $d(t)$ and distance to the closest physical point $d_{c}(t)$.

[4] B. Damas and J. Santos-Victor, "Avoiding moving obstacles: the forbidden velocity map," in 2009 IEEE/RSJ International Conference on Intelligent Robots and Systems, Oct 2009, pp. 4393-4398.

[5] J. van den Berg, J. Snape, S. J. Guy, and D. Manocha, "Reciprocal collision avoidance with acceleration-velocity obstacles," in 2011 IEEE International Conference on Robotics and Automation, May 2011, pp. 3475-3482.

[6] Z. Shiller, F. Large, and S. Sekhavat, "Motion planning in dynamic environments: obstacles moving along arbitrary trajectories," in Proceedings 2001 ICRA. IEEE International Conference on Robotics and Automation (Cat. No.01CH37164), vol. 4, 2001, pp. 3716-3721 vol.4.

[7] F. Large, C. Laugier, and Z. Shiller, "Navigation among moving obstacles using the NLVO : Principles and applications to Intelligent Vehicles," Autonomous Robots, vol. 19, no. 2, pp. 159-171, Sep. 2005, voir basilic : http://emotion.inrialpes.fr/bibemotion/2005/LLS05/ optkey: large-AR05. [Online]. Available: https://hal.inria.fr/inria00182105

[8] Y. Koren and J. Borenstein, "Potential field methods and their inherent limitations for mobile robot navigation," in Robotics and Automation,
1991. Proceedings., 1991 IEEE International Conference on, Apr 1991, pp. 1398-1404 vol.2.

[9] Y. Lu, Y. Yixin, and L. Cheng-Jian, "A new potential field method for mobile robot path planning in the dynamic environments," Asian Journal of Control, vol. 11, no. 2, pp. 214-225, 2009. [Online]. Available: https://onlinelibrary.wiley.com/doi/abs/10.1002/asjc. 98

[10] J. Ren, K. A. McIsaac, and R. V. Patel, "Modified newton's method applied to potential field-based navigation for nonholonomic robots in dynamic environments," Robotica, vol. 26, no. 1, p. 117127, 2008.

[11] O. Montiel, U. Orozco-Rosas, and R. Seplveda, "Path planning for mobile robots using bacterial potential field for avoiding static and dynamic obstacles," Expert Systems with Applications, vol. 42, no. 12, pp. 5177 - 5191, 2015. [Online]. Available: http://www.sciencedirect.com/science/article/pii/S0957417415001402

[12] Q. Zhang, S.-g. Yue, Q.-j. Yin, and Y.-b. Zha, "Dynamic obstacleavoiding path planning for robots based on modified potential field method," in Intelligent Computing Theories and Technology, D.-S. Huang, K.-H. Jo, Y.-Q. Zhou, and K. Han, Eds. Berlin, Heidelberg: Springer Berlin Heidelberg, 2013, pp. 332-342.

[13] A. S. Matveev, C. Wang, and A. V. Savkin, "Real-time navigation of mobile robots in problems of border patrolling and avoiding collisions," Robotics and Autonomous Systems, vol. 60, no. 6, pp. 769 - 788, 2012. [Online]. Available: http://www.sciencedirect.com/science/article/pii/S0921889012000346

[14] A. V. Savkin and C. Wang, "A simple biologically inspired algorithm for collision-free navigation of a unicycle-like robot in dynamic environments with moving obstacles," Robotica, vol. 31, no. 6, p. 9931001, 2013.

[15] C. Wang, A. S. Matveev, A. V. Savkin, T. N. Nguyen, and H. T. Nguyen, "A collision avoidance strategy for safe autonomous navigation of an intelligent electric-powered wheelchair in dynamic uncertain environments with moving obstacles," in 2013 European Control Conference (ECC), July 2013, pp. 4382-4387.

[16] D. Leca, V. Cadenat, T. Sentenac, A. Durand-Petiteville, F. Gouaisbaut, and E. L. Flecher, "Sensor-based obstacles avoidance using spiral controllers for an aircraft maintenance inspection robot," in (Forthcoming) 2019 European Control Conference (ECC), 2019.

[17] M. Futterlieb, V. Cadenat, and T. Sentenac, "A navigational framework combining visual servoing and spiral obstacle avoidance techniques," in Informatics in Control, Automation and Robotics (ICINCO), 2014 11th International Conference on, vol. 02, Sept 2014, pp. 57-64.

[18] E. L. Flecher, A. Durand-Petiteville, V. Cadenat, T. Sentenac, and S. Vougioukas, "Design of a sensor-based controller performing u-turn to navigate in orchards," in International Conference on Informatics in Control, Automation and Robotics, Madrid, Spain, July 2017.

[19] K. N. Boyadzhiev, "Spirals and conchospirals in the flight of insects," The College Mathematics Journal, vol. 30, no. 1, pp. pp. 23-31, 1999. [Online]. Available: http://www.jstor.org/stable/2687199

[20] A. Mcfadyen, L. Mejias, and P. Corke, "Visual servoing approach to collision avoidance for aircraft," in 28th Congress of the International Council of the Aeronautical Sciences 2012, Brisbane Convention \& Exhibition Centre, Brisbane, QLD, September 2012. [Online]. Available: http://eprints.qut.edu.au/54328/

[21] A. Mcfadyen, P. Corke, and L. Mejias, "Rotorcraft collision avoidance using spherical image-based visual servoing and single point features," in Intelligent Robots and Systems (IROS), 2012 IEEE/RSJ International Conference on, Oct 2012, pp. 1199-1205.

[22] A. Isidori, Nonlinear control systems. Springer Science \& Business Media, 2013.

[23] L. Rokach and O. Maimon, Data mining and knowledge discovery handbook. Springer US, 2005, ch. Clustering methods, pp. 321-352. 